\title{
A Preliminary Study of Constructing Tissue Engineered Oral Mucosa
}

\author{
Qin Zhang*, Yu-Jing Tian, Tao Ding, and De-Hong Feng \\ Wuxi People's Hospital Affiliated Nanjing Medical University, Wuxi, Jiangsu Province, PR China
}

*Corresponding author: Qin Zhang, No. 299, Qing Yang Road, Wuxi People's Hospital Affiliated Nanjing Medical University, Wuxi, Jiangsu Province, 214023, PR China, Tel: +86-0510-85351111; E-mail: zhangqin993@sina.com

Received: 04 Aug, 2021 | Accepted: 11 Aug, 2021 | Published: 19 Aug, 2021

Citation: Zhang Q, Tian YJ, Ding T, Feng DH (2021) A Preliminary Study of Constructing Tissue Engineered Oral Mucosa. Int J Dent Oral Health 7(5): dx.doi.org/10.16966/2378-7090.377

Copyright: (C) 2021 Zhang Q, et al. This is an open-access article distributed under the terms of the Creative Commons Attribution License, which permits unrestricted use, distribution, and reproduction in any medium, provided the original author and source are credited.

\begin{abstract}
Adipose derived stem cells (ADSCs) have a great potential for tissue-engineering purposes, and they may be introduced in oral mucosa tissue engineering for urethroplasty. This study was aimed to develop a tissue-engineered oral mucosa through seeding oral keratinocytes (OKs) and adipose derived stem cells (ADSCS) on small intestine submucosa (SIS). From August 2018 to October 2019, an observational study was conducted in the laboratory of our hospital, to develop a tissue-engineered oral mucosa.SIS was obtained from porcine small intestine, and OKs and ADSCs were obtained from canine sources and were cultured and expanded in vitro. The two cell lines were seeded on the two surfaces of the SIS, and the cell-scaffold compound graft was cultured in an air fluid level for 1 week. The SIS exhibited a porous membrane, and no cells were found through HE staining. The model cultured with OK-SIS only formed a thin and loose epithelium. Whereas the model cultured with OK-SIS-ADSC was much thicker and denser. The co-cultured of ADSC and OK grew well on the SIS in which the OKs formed a multilayer of epithelium. So it is feasible to construct a tissue-engineered oral mucosa graft with ADSCS, OKs and SIS. The ADSCs contribute to a thicker epithelium formation.
\end{abstract}

Keywords: Tissue engineering; Adipose derived stem cells; Oral keratinocytes; Urethroplasty

\section{Introduction}

The treatment of complex urethra stricture or urethral defect remains one of the most difficult problems in clinical urology, and tissue engineering seems to be the best choice for urethroplasty. Acellular matrices and cell-seeded constructs have been used in the clinical treatment of urethra stricture [1-3], and OK-seeded acellular matrices were demonstrated to work well in an animal urethroplasty study [4]. Another study has revealed that adipose derived stem cells (ADSCs) enhance angiogenesis under ischemic conditions, secrete angiogenic and antiapoptotic cytokines, express markers of vessel generation, and can actually differentiate into endothelial cells [5]. Thus, the introduction of ADSCs in tissue engineering may accelerate the angiogenesis process of the graft. In our previous study, we found that the existence of ADSCs could activate the proliferation and migration rate of oral keratinocytes (OKs). Therefore, in the present study, we cultured OKs and ADSCs and then seeded them onto acellular small intestine submucosa (SIS) to obtain a new type of tissue-engineered tissue for urethroplasty.

\section{Material and Methods}

This is an observational study that tries to make a tissue-engineered oral mucosa through seeding oral keratinocytes (OKs) and adipose derived stem cells (ADSCs) on small intestine submucosa (SIS).
From August 2018 to October 2019, we complete this study in the laboratory of our hospital.

\section{Scaffold preparation}

Porcine small intestine tissues were harvested from a recently sacrificed white pig weighing $70 \mathrm{~kg}$. The mucosa and the muscularis of the small intestine were scraped bluntly to obtain the SIS tissue. To induce cell lysis, the isolated matrices were stirred for 14 days at $37^{\circ} \mathrm{C}$ using $1 \%$ Triton X100(Sigma) and 0.1\% (v/v) ammonium hydroxide. HE staining was used to identify whether all of the cells were removed from scaffolds. The scaffolds were then freeze dried, packaged and irradiated with $\mathrm{Co}^{60}$ at a dose of $35 \mathrm{kGy}$ for sterilization. The obtained SIS scaffolds were stored at $4^{\circ} \mathrm{C}$ before use.

\section{Oral keratinocyte $(\mathrm{OK})$ culture}

The whole animal experimental protocol was approved by the Animal Care and use Committee at our institution before the initiation of the study. A $3 \mathrm{~cm} \times 1 \mathrm{~cm}$ piece of buccal mucosa was harvested from the lower lip of a 1.5-year-old Beagle under intravenous anesthesia with remifentanil and propofol, and the wound was sutured with 4-0 silk sutures. Antibiotics were administrated intravenously for 4 days postoperatively to prevent infection. The mucosa then was transferred to sterile phosphate buffered solution (PBS), and a modification of the Rheinwald and Green method [6] for cutaneous keratinocytes 
was used to isolate and culture the oral keratinocytes. The buccal mucosa was digested with $0.2 \mathrm{U} / \mathrm{ml}$ dispase II enzyme, and the dermis was removed. The oral keratinocytes were then isolated from the epidermis after trypsinization and were seeded onto a feeder layer of 3T3 mouse fibroblasts inhibited by mitomycin (i3T3) in Dulbecco's Modified Eagle's Medium(DMEM, Gibco, USA ) supplemented with $10 \%$ fetal bovine serium (FCS) and keratinocyte serum free medium (KSFM, Gibco, USA) at a 1:1 ratio. A portion of the OKs were seeded into an empty tissue culture dish and cultured in KSFM medium for comparison. For expansion, the OKs were cultured and re-fed every 3 days until they were $85-95 \%$ confluent. The residual i3T3 cells were removed with dispase II before the keratinocytes were detached using $0.2 \mathrm{U} / \mathrm{ml}$ trypsin.

\section{Adipose-derived stem cell (ADSC) culture}

A piece of adipose tissue approximately $3 \mathrm{~cm} \times 2 \mathrm{~cm} \times 1 \mathrm{~cm}$ in size was dissected from the subcutaneous inguinal ring of the same Beagle as above through a $3 \mathrm{~cm}$ longitudinal incision in the right groin area, which was intermittently sutured with 3-0 silk sutures after the harvest. The adipose tissue was transferred to sterile PBS. The visible small blood vessels were removed with eye scissors, and the remaining tissue was cut into small pieces and digested in $0.1 \%$ collagenase I (Worthington, USA) at $37^{\circ} \mathrm{C}$ for $1 \mathrm{~h}$, with uninterrupted shaking. The adipose derived stem cells (ADSCs) were harvested after filtration and centrifugation. The isolated ADSCs were then cultured in DMEM supplemented with $10 \% \mathrm{FBS}$ at $37^{\circ} \mathrm{C}$ with $5 \% \mathrm{CO}_{2}$ [7]. Upon reaching $80-90 \%$ confluence, the isolated cells were trypsinized, counted, and subcultured at a density of $10^{5}$ cells/100-mm dish.

\section{Observation and identification of OKs and ADSCs}

The cultured P3 OKs were identified using an immunofluorescence staining method. To determine the expression of cytokeratin AE1/ AE3, mouse monoclonal cytokeratin AE1/AE3 antibodies (SigmaAldrich) was used; this antibody was also used in flow cytometry to measure the purity of the OKs.

The cultured P5 ADSCs were identified by flow cytometry using antibodies against CD34, CD44, CD45, CD90, CD105 and CD106 obtained from BD Biosciences (Franklin Lakes, NJ, and USA).

\section{Cell seeding}

Before seeding, the SIS was soaked for 15 minutes in sterile saline for rehydrating, then immersed in sterile PBS for 48 hours and DMEM supplemented with 10\% FBS for another 48 hours. The ADSCs were gathered at the fifth cellular passage and seeded the high concentration cell suspension onto the SIS at a density of $3 \times 10^{6} / \mathrm{cm}^{2}$, and then the graft was incubated for 4 hours at $37^{\circ} \mathrm{C}$ with $5 \% \mathrm{CO}_{2}$ for cell adhesion before enough medium was added. The ADSC-seeded SIS was cultured for 4 days (during which the medium was changed every day) and transferred to a stainless steel wire mesh immersed in DMEM, with the cell-seeded surface facing the mesh and the nonseeded surface just on the air fluid level. The P3 OKs were collected and seeded onto the non-seeded surface at a density of $3 \times 10^{6} / \mathrm{cm}^{2}$. Similarly, the compound was incubated for 4 hours for cell adhesion before the KSFM was added. The compound was cultured in a mixed medium (DMEM:KSFM=1:1), which was changed every day, at an air fluid level for 7 days and then analyzed. Additionally, the third-passage OKs were seeded onto the SIS at a similar density without the ADSCs for comparison.

\section{Results}

The mouth wound did not affect the food consumption of the
Beagle, nor did the groin wound influence the mobility of the animal. The wounds healed without any signs of infection after 6 days for the mouth wound and 10 days for the groin wound.

After the cells were removed, the SIS presented as a porous membrane. The effect of acellularization was conformed through random staining with HE (Figure 1A) and Masson (Figure 1B), and no cells were visible. By the Masson staining (Figure 1B), we found that the SIS was consisted of uniform collagen fibers.

The OKs seeded onto the feeder layer of i3T3 were polygonal and exhibited a characteristic cobblestone-like morphology (Figure 1C). After 8 days, 90\% coverage of the culture plate was achieved, and the cells could be passaged for 8 generations. The total amount of OKs at passage 3 was $5 \times 10^{7}$, which was sufficient for seeding on SIS at a density of $3 \times 10^{6} / \mathrm{cm}^{2}$. Without the feeder layer of i3T3, nearly 2 weeks passed before the OKs covered $90 \%$ of the culture plate; these cells could only be subcultured for 2 generations before aging and had multiple shapes and sizes (Figure 1D). Immunofluorescence staining indicated cytokeratin AE1/AE3 expression in the OKs (Figure 1E), and the flow cytometry results indicated that the expression rate of cytokeratin AE1/AE3 in the passage 3 OKs was $90.25 \%$.

After incubation for nearly 7 days, the ADSC population covered approximately $90 \%$ of the culture plate; these cells were fusi form and exhibited a spindle-shaped morphology (Figure 1F) and could subcultured for 10 generations without signs of aging. Flow cytometry analysis demonstrated the expression of CD90 (95.53\%), CD34 (40.20\%), CD44 (97.42\%) and CD105 (65.25\%) in the ADSCs, whereas there was no significant expression of CD45 (2.13\%) or CD106 (2.05\%)

The passage- 5 ADSCs and passage- 3 OKs cultured with the i3T3 cells were isolated and seeded on the two surfaces of SIS, respectively, at a density of $3 \times 10^{6} / \mathrm{cm}^{2}$ for each cell line. The compound grafts were cultured with the OK-seeded surface upwards in an air fluid level for 1 week. Through scanning electron microscopy, we found that both the ADSCs (Figure 2) and OKs (Figure 3) adhered tightly to the SIS. Both of the two cell lines stretched out pseudopodia peripherally and connected with each other. Through random HE staining, we found that the model cultured with OK-SIS only formed a thin and loose epithelium (Figure 4A). Whereas the model cultured with OK-SISADSC was much thicker and denser (Figure 4B). The co-cultured of ADSC and OK grew well on the SIS in which the OKs formed a multilayer of epithelium.

\section{Discussion}

Urethral strictures or defects induced by different causes are common conditions in urology, and substitution urethroplasty is usually needed in many cases. Although many surgical methods for performing urethroplasty have emerged in recent years, complex urethra stricture or defects remain the most challenging problems in urologic surgery. Buccal, lingual, bladder and colonic mucosa and genital or extragenital skin are the major elements used in urethroplasty, especially the former two. However, the limited amount of autologous donor tissue limits these types of reconstructions. Moreover, complications, such as pain, swelling, numbness, tightness and motor defects of the mouth still occur during long-term follow-up [8-10].

Tissue engineering combines the principles and methods of the life sciences with those of engineering to develop an approach to repair damaged tissue and replace entire tissue structures. During the last decade, the use of biodegradable materials seeded with cells for urogenital reconstruction has been increasingly common [2,3,11]. 


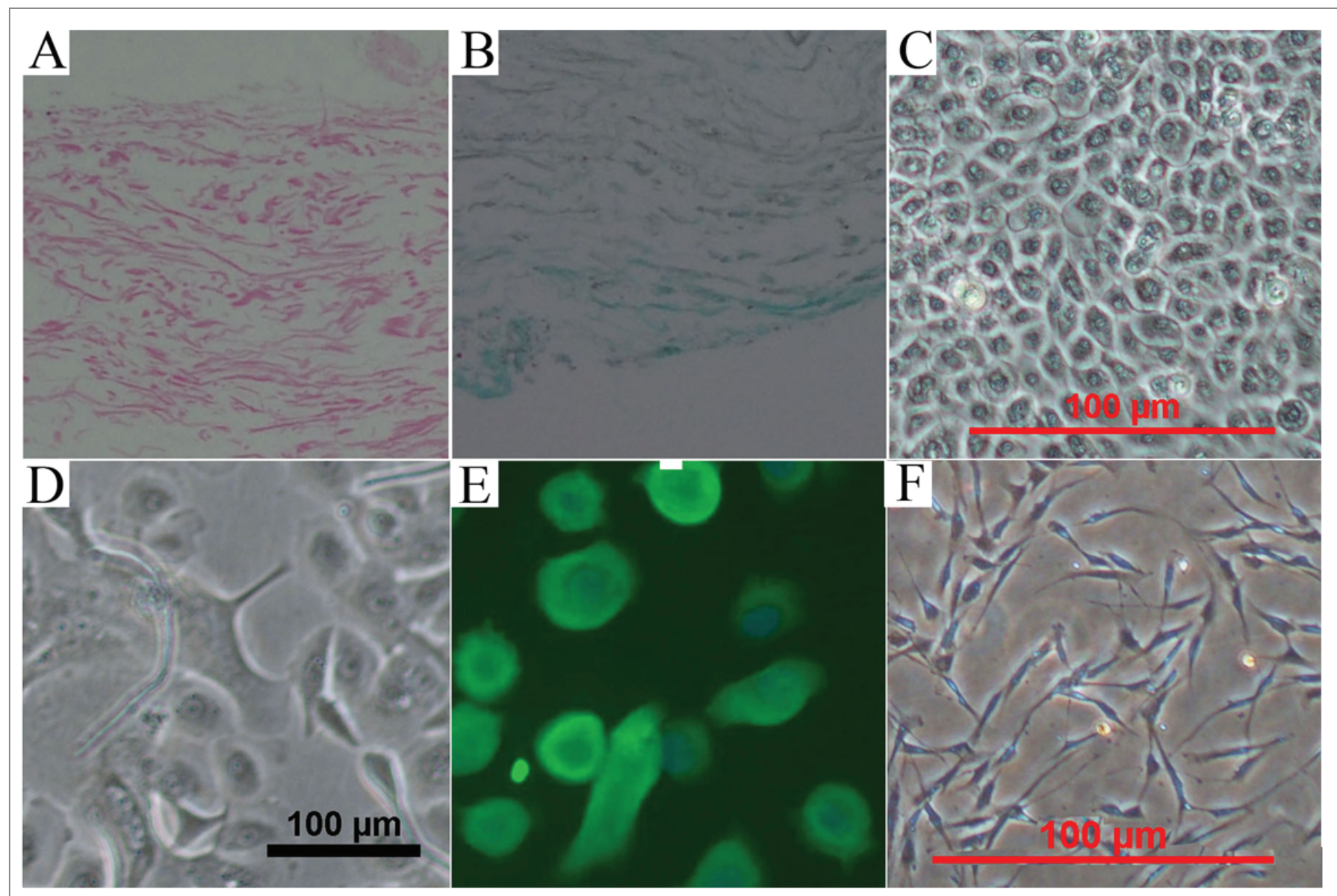

Figure 1: A. HE staining of SIS; B. Masson staining of SIS; C. The OKs exhibit a characteristic cobblestone-like morphology; D. Without the i3T3 feeder layer, the P2 OKs aged and exhibited multiple shapes and sizes; E. Immunofluorescence staining verifying cytokeratin AE1/AE3 expression in the OKs. Cell nuclei are stained with Hoechst; $F$. The ADSCs were fusi form and exhibited spindle-shaped morphologies.

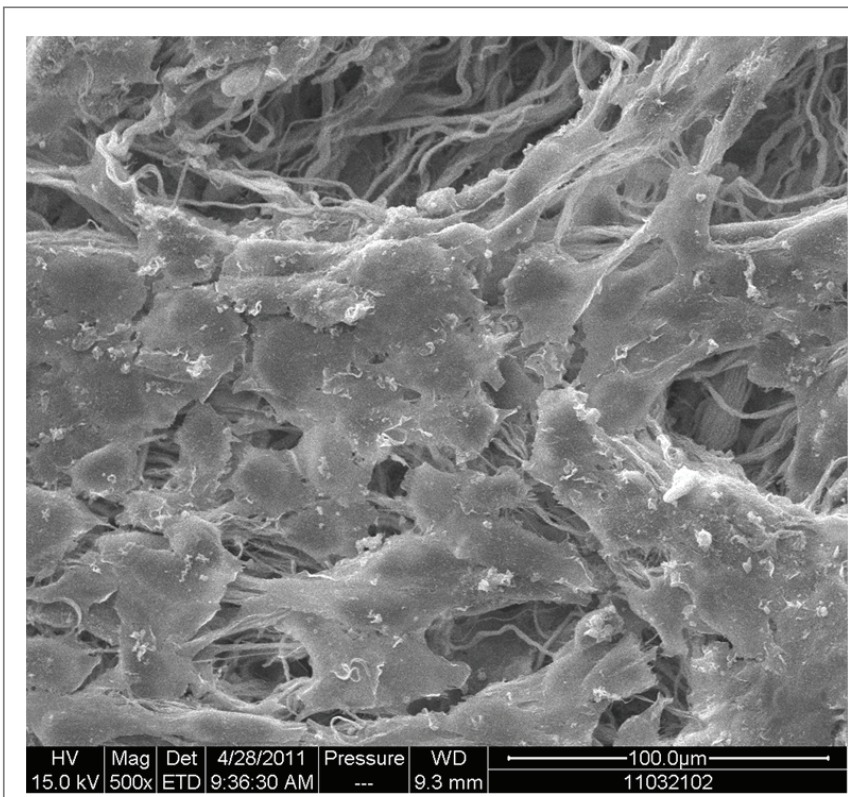

Figure 2: The OKs survive well on SIS, as shown by scanning electron microscopy.

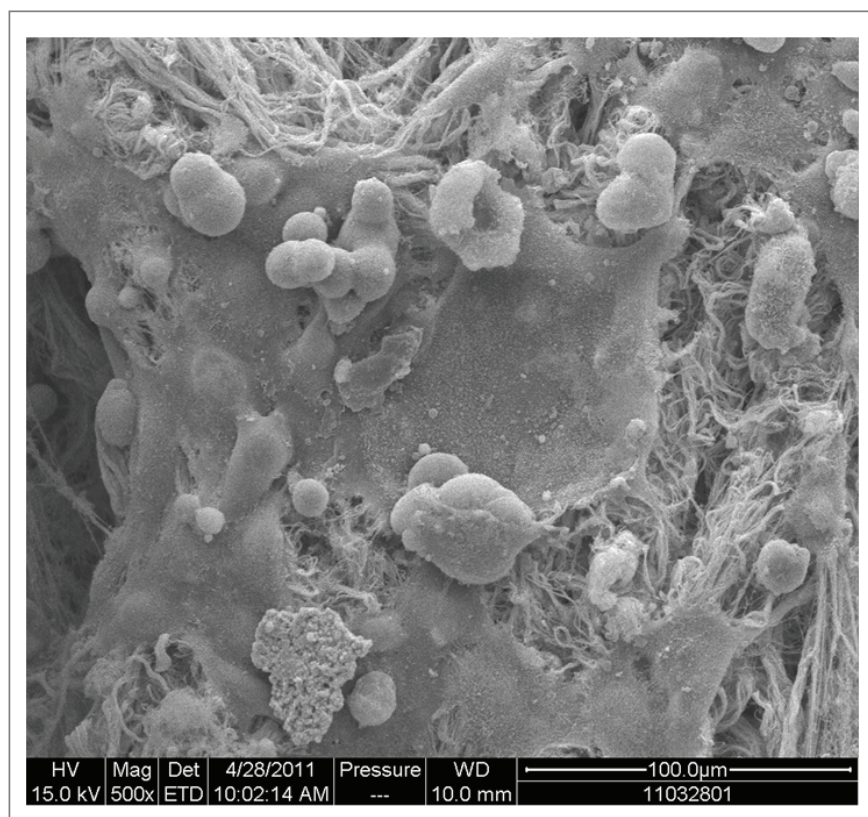

Figure 3: The ADSCs survive well on SIS, as shown by scanning electron microscopy. 

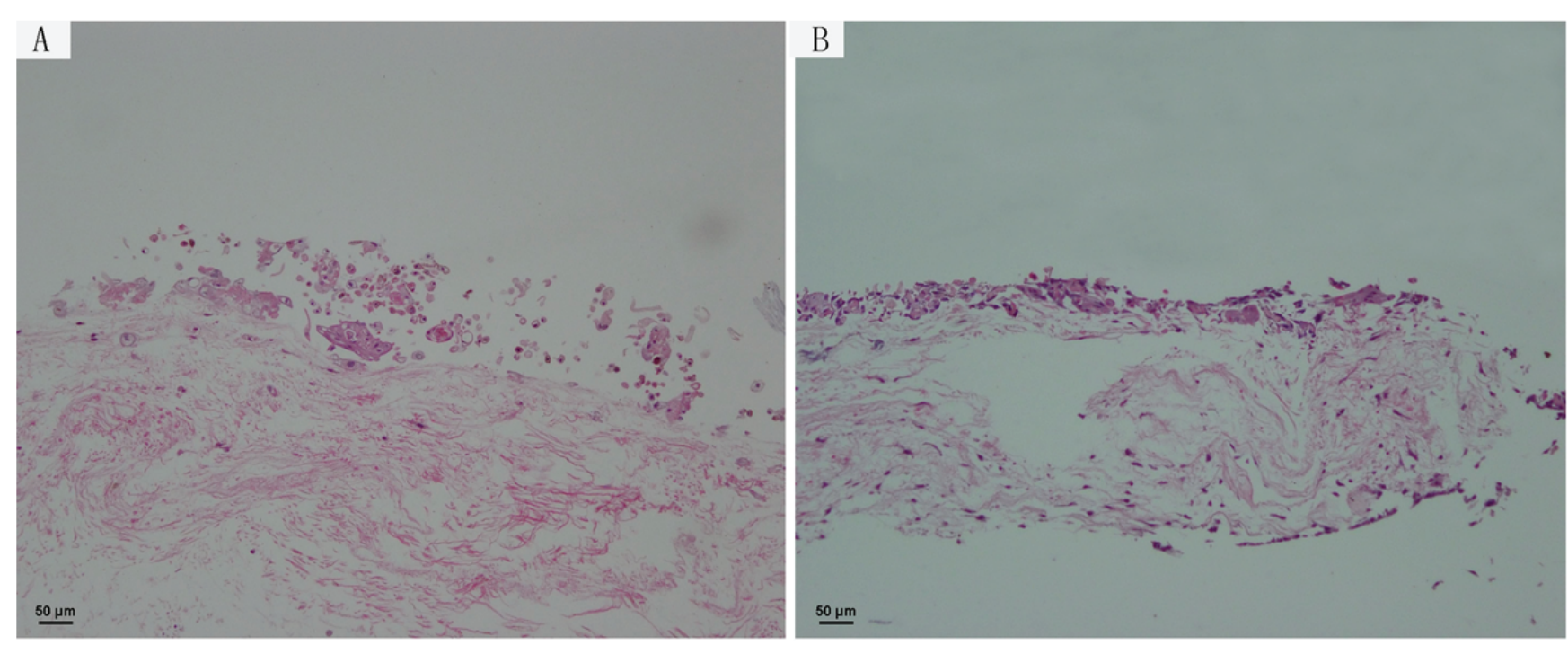

Figure 4: The epithelium formed by different cell-scaffold constructs: A. OK-SIS; B. OK-SIS-ADSC.

The aim of this study was to undertake a pilot evaluation of the construction of tissue-engineered oral mucosa (TEOM) with OKs, ADSCs and SIS. We isolated canine OKs and ADSCs, cultured and expanded them in vitro, and developed a TEOM model by seeding the two cell lines separately on the two sides of sterilized porcine SIS. Our goal was to provide a customized replacement tissue for urethroplasty.

The cell culture technique used in this study proved reliable. The OKs seeded onto the feeder layer of i3T3 cells demonstrated strong proliferation, and it was relatively simple to obtain an adequate number of good-quality OKs. The ADSCs also showed strong proliferation, and we obtained a sufficient quantity of cells for seeding. After 21 days culture and amplification, we obtained approximately $5 \times 10^{7}$ OKs of passage 3 and approximately $2 \times 10^{8}$ ADSCs of passage 5 . If all of the cultured cells were used for the preparation of the TEBM, it would have been possible to construct a strip of $3 \mathrm{~cm} \times 5 \mathrm{~cm}$. If a larger strip were necessary, continued culturing and expanding of both of the two cell lines would produce an adequate number. In our study, the OKs seeded on i3T3 could be passaged for 6 generations, and the ADSCs could be passaged for more than 10 generations; thus, it is realistic to obtain a sufficient amount of OKs and ADSCs.

A suitable scaffold biomaterial is a major challenge in developing tissue-engineered materials for urethroplasty. This biomaterial must be biocompatible to minimize any inflammatory or foreign-body response once implanted; it should also encourage cell adhesion, proliferation, migration, and differentiation to facilitate and prompt the development of new tissue. Furthermore, once implanted, it should degrade automatically after maturation of the new tissue. SIS has all of these qualities, and it has been previously used in urologic surgery for urethroplasty [1]. In our investigation, we successfully decelled the submucosa of porcine small intestine to gain the SIS. The SIS was retained on the relatively complete extracellular matrix-supporting frame, and the cell component was removed with antigen such that no inflammation mediated by immunoreaction could be induced. In addition, the SIS has a porous constitution and contains affluent collagen fibers, which are the results of the sticking and growth of cells. We found that both the ADSCs and OKs grow well on the SIS through scanning electron microscopy, and both of the cell lines peripherally extended pseudopodia and connected with each other (Figures 2,3).
Adipose tissue is derived from embryonic mesodermal precursors and contains multipotent progenitor cells capable of differentiating into mesenchymal tissue, such as chondrocytes, osteocytes, cardiomyocytes, neurons, and smooth muscle cells [12]. These progenitor cells are named adipose derived stem cells (ADSCs). The harvest of ADSCs is less difficult than any other type of adult stem cell, and the abundance of stem cells in adipose tissue is 100-1,000 times more than the stem cells in the same volume of bone marrow [13]. There has been a report demonstrating that ADSCs are immunoprivileged both in vitro and in vivo [14], enabling their use as universal donor cells for allogenic transplant. In previous reports [15], ADSCs exhibited wound healing and anti-oxidant effects that were attributed to the production and secretion of growth factors, such as bFGF, KGF, TGF- $\beta$, HGF and VEGF. Through a paracrine mechanism, these growth factors may stimulate both collagen synthesis and the migration of dermal fibroblasts and activate dermal fibroblasts and keratinocytes, resulting in accelerated vascularization, then accelerating wound healing in animal models [16]. There are also reports demonstrating that the ADSCs could accelerate the vascularization of tissue-engineered grafts used in the repair of skin defects [17]. Thus, we expect that the ADSCs may accelerate the formation of new mucosa through activating the keratinocytes in the oral mucosa tissue and also accelerate the vascularization of the tissue-engineered oral mucosa.

It is difficult to differentiate the ADSCs from fibroblasts based solely on their morphology in culture, as both of them are fusiform and exhibit a spindle-shaped morphology. In this study, flow cytometry analysis demonstrated that the canine-derived ADSCs expressed high levels of stem cell-related antigens (CD90, CD44, CD34, and CD105), but did not express CD45 or CD106. Our results are similar to previous reports [18], suggesting that our population of ADSCs are mesenchymal stem cells.

Similar to urethral epithelial cells, oral keratinocytes (OKs) are considered cover epithelium, having a thick epithelium adapted to a wet environment and easy to harvesting. Thus, tissue-engineered oral mucosa holds a great promise in urethroplasty. In an animal study in our laboratory, the tissue-engineered oral mucosa constructed by the OKs seeded on bladder acellular matrix was used in urethroplasty [4]. It was also reported that tissue-engineered oral mucosa was used in a 
urology surgery clinic for urethroplasty, although the results were not satisfactory [2].

In this study, we successfully isolated and expanded OKs in vitro. The OKs were polygonal and exhibited a characteristic cobblestone-like morphology (Figure 1C). Flow cytometry and immunofluorescence staining (Figure 1E) indicated the expression of cytokeratin AE1/AE3, supporting our claims that we had, in fact, isolated and expanded the keratinocytes.

In a previous study in our laboratory [4,7], OKs were seeded on a biomaterial scaffold, and then the compound graft was cultured in an air-fluid level for 1 week to construct a tissue-engineered oral mucosa graft. As fully differentiated cells, the OKs were cultured and expanded on the feeder layer of i3T3 cells, without which the OKs would rapidly age. Because the OKs were seeded on the scaffold and later used in vivo for urethroplasty, they may rapidly age after leaving the feeder layer of i3 3 , which may slow the formation of new mucosa. In our previous study, we found that the existence of ADSCs could activate OKs: the proliferation of OKs was increased and their migration was enhanced in a coculture environment with ADSCs. Thus, in the present study, we seeded OKs on one surface of SIS and ADSCs on the other, to make a 3-D culture environment [19]. In addition, we seeded only OKs on the SIS as control. After culture in an air fluid level for 1 week, the cell-graft compound grafts were analyzed. HE staining demonstrated that epithelium was formed on the OKs-seeded surface and that the thickness of the epithelium on the OK-SIS-ADSC compound graft was thicker than that on the OK-SIS compound graft (Figure 4). Furthermore, the epithelium morphology of the former was better than that of the latter, which may be attributed to the function of the ADSCs because the OKs seeded per unit area were of equal number and this function may continue in vivo.

In this study, we successfully construct a tissue-engineered oral mucosa with ADSCs, OKs and SIS. Our results demonstrated that the existence of ADSCs could help in epithelium formation in vitro. In the future, we will use this OK-SIS-ADSC compound graft for urethroplasty in an animal study.

\section{Conclusion}

It is feasible to construct a tissue-engineered oral mucosa graft with OKs and ADSCs seeded on SIS. The existence of ADSCs is conducive to the formation of new epithelium.

\section{Declaration of Interest}

The authors confirm that they have no conflicts of interest.

\section{References}

1. Palminteri E, Berdondini E, Colombo F, Austoni E (2007) Small intestine submucosa (SIS) graft urethroplasty: short-term results. Eur Urol 51: 1695-1701.

2. Bhargava S, Patterson JM, Inman RD, MacNeil S, Chapple CR (2008) Tissue-engineered buccal mucosa urethroplasty-clinical outcomes. Eur Urol 53: 1263-1271.

3. Raya-Rivera A, Esquiliano DR, Yoo JJ, Lopez-Bayghen E, Soker S, et al. (2011) Tissue-engineered autologous urethras for patients who need reconstruction: an observational study. Lancet 377: 11751182.

4. Li C, Xu YM, Song LJ, Fu Q, Cui L, et al. (2008) Urethral reconstruction using oral keratinocyte seeded bladder acellular matrix grafts. J Urol 180: $1538-1542$.
5. Kim EK, Li G, Lee TJ, Hong JP (2011) The effect of human adiposederived stem cells on healing of ischemic wounds in diabetic nude mouse model. Plast Reconstr Surg 128: 387-394.

6. Rheinwald JG, Green H (1975) Serial cultivation of strains of human epidermal keratinocytes: the formation of keratinizing colonies from single cells. Cell 6: 331-343.

7. Li C, Xu Y, Song L, Fu Q, Cui L, et al. (2008) Preliminary experimental study of tissue-engineered urethral reconstruction using keratinocytes seeded on BAMG. Urol Int 81: 290-295.

8. Xu YM, Qiao Y, Sa YL, Wu DL, Zhang J, et al. (2004) 1-stage urethral reconstruction using colonic mucosa graft for the treatment of a long complex urethral stricture. J Urol 171: 220-223.

9. Kamp S, Knoll T, Osman M, Häcker A, Michel MS, et al. (2005) Donorsite morbidity in buccal mucosa urethroplasty: lower lip or inner cheek? BJU Int 96: 619-623.

10. Raber M, Naspro R, Scapaticci E, Salonia A, Scattoni V, et al. (2005) Dorsal onlay graft urethroplasty using penile skin or buccal mucosa for repair of bulbar urethral stricture: results of a prospective single center study. Eur Urol 48: 1013-1017.

11. Atala A, Bauer SB, Soker S, Yoo JJ, Retik AB (2006) Tissue-engineered autologous bladders for patients needing cystoplasty. Lancet 367 : 1241-1246.

12. Zuk PA, Zhu M, Ashjian P, De Ugarte DA, Huang Jl, et al. (2002) Human adipose tissue is a source of multipotent stem cells. Mol Biol Cell 13: 4279-4295.

13. Rangappa S, Fen C, Lee EH, Bongso A, Sim EK (2003) Transformation of adult mesenchymal stem cells isolated from the fatty tissue into cardyoiomcytes. Ann Thorac Surg 75: 775-779.

14. Rodriguez AM, Pisani D, Dechesne CA, Turc-Carel C, Kurzenne JY, et al. (2005) Transplantation of a multipotent cell population from human adipose tissue induces dystrophin expression in the immunocompetent mdx mouse. J Exp Med 201: 1397-1405.

15. Kim WS, Park BS, Sung JH (2009) The wound-healing and antioxidant effects of adipose-derived stem cells. Expert Opin Biol Ther 9: 879887.

16. Pries AR, Mulvany MJ, Bakker EN (2008) MBEC special issue on microcirculation "engineering principles of vascular networks". Med Bio Eng Comput 46: 407-409.

17. Liu S, Zhang $H$, Zhang $X$, Lu W, Huang $X$, et al. (2011) Synergistic angiogenesis promoting effects of extracellular matrix scaffolds and adipose-derived stem cells during wound repair. Tissue Eng part $A$ 17: 725-739.

18. Mitchell JB, McIntosh K, Zvonic S, Garrett S, Floyd ZE, et al. (2006) Immunophenotype of human adipose-derived cells: temporal changes in stromal-associated and stem cell-associated markers. Stem cells 24: 376-385.

19. Linder P, Trzewik J, Rüffer M, Artmann GM, Digel I, et al. (2010) Contractile tension and beating rates of self-exciting monolayers and 3D-tissue constructs of neonatal rat cardiomyocytes. Med Bio Eng Comput 48: 59-65. 